\title{
Local Correction with Constant Error Rate
}

\author{
Noga Alon* Amit Weinstein ${ }^{\dagger}$
}

August 5, 2018

\begin{abstract}
A Boolean function $f$ of $n$ variables is said to be $q$-locally correctable if, given a blackbox access to a function $g$ which is "close" to an isomorphism $f_{\sigma}(x)=f_{\sigma}\left(x_{1}, \ldots, x_{n}\right)=$ $f\left(x_{\sigma(1)}, \ldots, x_{\sigma(n)}\right)$ of $f$, we can compute $f_{\sigma}(x)$ for any $x \in \mathbb{Z}_{2}^{n}$ with good probability using $q$ queries to $g$. It is known that degree $d$ polynomials are $O\left(2^{d}\right)$-locally correctable, and that most $k$-juntas are $O(k \log k)$-locally correctable, where the closeness parameter, or more precisely the distance between $g$ and $f_{\sigma}$, is required to be exponentially small (in $d$ and $k$ respectively).

In this work we relax the requirement for the closeness parameter by allowing the distance between the functions to be a constant. We first investigate the family of juntas, and show that almost every $k$-junta is $O\left(k \log ^{2} k\right)$-locally correctable for any distance $\varepsilon<0.001$. A similar result is shown for the family of partially symmetric functions, that is functions which are indifferent to any reordering of all but a constant number of their variables. For both families, the algorithms provided here use non-adaptive queries and are applicable to most but not all functions of each family (as it is shown to be impossible to locally correct all of them).

Our approach utilizes the measure of symmetric influence introduced in the recent analysis of testing partial symmetry of functions.
\end{abstract}

\section{Introduction}

Local correction of functions deals with the task of determining the value of a function in a given point by reading its values in several other points. More precisely, we care about locally correcting specific functions which are known up to isomorphism, that is, functions which are known up to reordering of the input variables. Our main interest is identifying the number of needed queries for this task, for a given function. For a permutation $\sigma \in \mathcal{S}_{n}$ and a function $f=f\left(x_{1}, \ldots, x_{n}\right): \mathbb{Z}_{2}^{n} \rightarrow$ $\mathbb{Z}_{2}$, let $f_{\sigma}$ denote the function given by $f_{\sigma}\left(x_{1}, \ldots, x_{n}\right)=f\left(x_{\sigma(1)}, \ldots, x_{\sigma(n)}\right)$.

Question. Given a specific Boolean function $f$, what is the needed query complexity in order to correct an input function which is close to some isomorphism $f_{\sigma}$ of $f$ ?

This question can be seen as a special case of locally correctable codes (see, e.g., [13]). Each codeword would be the $2^{n}$ evaluations of an isomorphic copy of the input function, and thus the

*Sackler School of Mathematics and Blavatnik School of Computer Science, Tel Aviv University, Tel Aviv 69978, Israel. Email: nogaa@tau.ac.il. Research supported in part by an ERC Advanced grant, by a USA-Israeli BSF grant and by the Israeli I-Core program.

${ }^{\dagger}$ Blavatnik School of Computer Science, Tel Aviv University, Tel Aviv 69978, Israel. Email: amitw@tau.ac.il. Research supported in part by an ERC Advanced grant and by the Israeli Centers of Research Excellence (I-CORE) program. 
number of distinct codewords is at most $n$ !, and we would like to correct any specific value of the given noisy codeword using as few queries as possible.

The notion of closeness in the above question plays a crucial role in answering it. We say that two functions are $\varepsilon$-close to one another if they differ on at most an $\varepsilon$ fraction of the inputs. Equivalently, $f$ is $\varepsilon$-close to $f^{\prime}$ if $\operatorname{Pr}_{x}\left[f(x) \neq f^{\prime}(x)\right] \leq \varepsilon$, over a uniformly chosen $x \in \mathbb{Z}_{2}^{n}$. The main focus of this work is to better understand the functions for which a constant number of queries suffices for local correction, while we allow $\varepsilon$ to be a constant as well. In particular, we show that for partially symmetric functions, that is, functions which are symmetric with respect to all but a constant number of their variables, this is typically the case.

The field of local correction of Boolean functions is closely related to that of property testing, and in particular to testing isomorphism of functions. In testing, the goal is to distinguish between a function which satisfies some property and functions which are far from satisfying that property, while here we are guaranteed the input function is close to satisfy a property, the property of being isomorphic to some specific function, and we are required to locally correct a given input. Due to this resemblece, many tools used in the research of local correction are borrowed from the field of testing functions isomorphism and property testing in general (see e.g. [9, 11, 2, 17, 1, 10]).

\section{$1.1 \quad$ Preliminaries}

Below is the formal definition of locally correctable functions, as given in [4].

Definition. A Boolean function $f: \mathbb{Z}_{2}^{n} \rightarrow \mathbb{Z}_{2}$ is q-locally correctable for $\varepsilon>0$ if the following holds. There exists an algorithm that given an input function $g$ which is $\varepsilon$-close to an isomorphism $f_{\sigma}$ of $f$, can determine the value $f_{\sigma}(x)$ for any specific $x \in \mathbb{Z}_{2}^{n}$ with probability at least $2 / 3$, using $q$ queries to $\mathrm{g}$.

The two interesting parameters of the above definition are the noise rate $\varepsilon$ and the number of queries $q$. In our recent work 4 it is shown that when we allow the noise rate to be relatively small, depending on the structure of the function, functions from several interesting classes can be locally corrected. More precisely, it is observed that every degree $d$ polynomial can be locally corrected from noise rate of $\varepsilon<2^{-d-3}$ using $O\left(2^{d}\right)$ queries. The family of $k$-juntas, that is, functions which only depend on $k$ of their input variables, are in particular degree $k$ polynomials and hence the same upper bound is applicable. However, this is usually not tight as most $k$-juntas can actually be locally corrected from the same noise rate using $O(k \log k)$ queries. For more details on the above results, we refer the reader to 4 .

The above results require an exponential dependency between the noise rate $\varepsilon$ and the parameter determining the structure of the function, such as the size of the junta or the degree of the polynomial. This raises the following natural question. Can we locally correct functions from these families when the noise rate is higher or even constant? More generally, which functions can or cannot be locally corrected with a constant number of queries from a given constant noise rate?

\subsection{Our results}

The main result of this work is the identification of two families, in which most functions can be locally corrected from a constant noise rate. The first family is that of juntas. We say that almost 
every $k$-junta satisfies a property if only $\varepsilon_{k}$ fraction of the $k$-juntas do not satisfy it, where $\varepsilon_{k}$ tends to zero as $k$ tends to infinity.

Theorem 1.1. Almost every $k$-junta can be locally corrected from a noise rate of $\varepsilon=0.001$, using $O\left(k \log ^{2} k\right)$ non-adaptive queries.

Similarly to the result in [4], this statement applies to almost every junta (and not to all of them). The main differences between the results are the constant noise rate which does not depend on the junta size $k$, and the fact that the algorithm we describe is non-adaptive (at the expense of increasing the query complexity by a logarithmic factor).

The second main result presented here is an extension of Theorem 1.1 to another family of functions, the family of partially symmetric functions, as defined in [8]. A function $f$ is called $t$-symmetric if there exists a set of $t$ variables such that $f$ is symmetric with respect to these variables (that is, any reordering of these variables does not change the function). To better see the connection between these functions and juntas, the following equivalent definition is often useful. We say that $f$ is $(n-k)$-symmetric if there exists a set of $k$ variables such that the output of $f$ is determined by these $k$ variables, and the Hamming weight of the others. A $k$-junta is in particular an $(n-k)$-symmetric function, and hence the following theorem can be viewed as a generalization of Theorem 1.1.

Theorem 1.2. Almost every $(n-k)$-symmetric function can be locally corrected from a noise rate of $\varepsilon=0.001$ using $O\left(k \log ^{2} k\right)$ non-adaptive queries.

Here too the term "almost every" means that only $\varepsilon_{n}$ fraction of these functions do not satisfy the above where $\varepsilon_{n}$ tends to zero as $n$ tends to infinity, and it does not depend on $k$.

The proof of the theorem relies on the analysis of partially symmetric functions and borrows some of the ideas provided in [8]. Notice that although juntas are in particular partially symmetric functions, Theorem 1.1 is not a corollary of Theorem [1.2, as juntas represent a small fraction of all partially symmetric functions, and these results are applicable only to most functions in the respective families.

The above theorems apply to almost every junta and partially symmetric function but not to all of them. As the next simple result indicates, this restriction is unavoidable. Some functions in these families are not locally correctable from a constant noise rate, regardless of the number of queries.

Proposition 1.3. For every constant $\varepsilon>0$, there exists $k(\varepsilon)$ such that the following holds. For every $k \geq k(\varepsilon)$, there exist $k$-juntas which cannot be locally corrected from $\varepsilon$ noise rate, regardless of the number of queries.

Proof. Fix some $\varepsilon>0$ and let $k(\varepsilon)=\lceil\log 1 / \varepsilon\rceil$. Given some $k \geq k(\varepsilon)$, we consider the $k$-junta $f$ which is defined to be 1 only when the first variable is 1 and the other $k-1$ variables following it are 0 . This function is obviously a $k$-junta as it is determined by the first $k$ variables only. Notice however that the constant zero function is $2^{-k} \leq \varepsilon$ close to $f$, and therefore if we try to locally correct it, we will not be able to identify which isomorphism of $f$ we were given. Hence we would not be able to locally correct $f$, regardless of the number of queries.

The above result is rather extreme, in the sense that we have no way of identifying which original isomorphism was chosen. For most functions this is not the case, and this allows us to 
achieve the previous results. The last result we present in this work shows that locally correcting most functions is relatively hard, even from the smallest possible error rate of a single error.

Theorem 1.4. Almost every function over $n$ variables cannot be locally corrected, even from a single error (i.e., $\varepsilon=2^{-n}$ ), using fewer than $n / 100$ non-adaptive queries.

The proof of Theorem 1.4 appears in Section 4 along with several open questions. In Sections 2 and 3 we prove Theorems 1.1 and 1.2, respectively. The two proofs share a similar structure.

\section{Correcting juntas}

Our approach for locally correcting juntas consists of two main steps. The goal of the first step is to identify the junta variables, i.e. those variables which determine the output of the function. Since our query complexity should not depend on the input size, one cannot hope to recover their exact location. Instead, we use the testing-by-implicit-learning approach (see, e.g., 12]) and only identify large sets which contain these variables. The second step, performed after we have identified $k$ sets, each of which containing one of the junta variables, is recovering their internal order (out of the $k$ ! possible orderings). Once both these steps are completed, we would be able to output the correct value of the function for the requested input.

In Section 2.1 we define some of the tools and typical properties of juntas. The two steps of the algorithm are later described in Secctions 2.2 and 2.3, completing the proof of Theorem 1.1 .

\subsection{Properties of juntas}

Since juntas depend on a relatively small number of variables, we often consider their concise representation over these variables only. Given a $k$-junta $f$, we denote the core of $f$ by $f_{\text {core }}: \mathbb{Z}_{2}^{k} \rightarrow$ $\mathbb{Z}$, which is the function $f$ restricted to its $k$ junta variables in their natural order.

A variable of a function is said to be influencing if modifying its value can modify the output of the function. Clearly a $k$-junta has at most $k$ influencing variables, which are in fact the junta variables (those which appear in its core). The following definition quantifies how influential a variable, or more generally a set of variables, is with respect to a given function.

Definition 2.1 (Influence). Given a Boolean function $f: \mathbb{Z}_{2}^{n} \rightarrow \mathbb{Z}_{2}$, the influence of a set of variables $J \subseteq[n]:=\{1,2, \ldots, n\}$ with respect to $f$ is defined by

$$
\operatorname{Inf}_{f}(J)=\operatorname{Pr}_{x, y}\left[f(x) \neq f\left(x_{\bar{J}} y_{J}\right)\right]
$$

where $x_{\bar{J}} y_{J}$ is the vector whose ith coordinate equals to $y_{i}$ if $i \in J$, and otherwise equals to $x_{i}$. When the set $J=\{i\}$ is a singleton, we simply write $\operatorname{Inf}_{f}(i)$.

An important property of influence is monotonicity. Namely, it is known (see, e.g., [11]) that for any two sets $J \subseteq K$ and any function $f, \operatorname{Inf}_{f}(J) \leq \operatorname{Inf}_{f}(K)$. Given this property, a set which has even a single variable with large influence must also have large influence. We heavily rely on this fact in our algorithm.

The result we present in this work is only applicable to most juntas. The following two propositions indicate two typical properties of juntas, which are required for our algorithm to work with 
high probability. The first, presented in Proposition 2.2, indicates that in a typical junta every influencing variable has constant influence. The second property bounds the distance between a typical junta and its isomorphisms, and is presented in Proposition 2.3. Notice that in both propositions, it suffices to consider the core of the junta rather than the entire function.

Proposition 2.2. Let $f: \mathbb{Z}_{2}^{k} \rightarrow \mathbb{Z}_{2}$ be a random core of a $k$-junta. Then with probability at least $1-2^{-\Omega(k)}$, any variable $i \in[k]$ out of the $k$ variables of $f$ has influence $\operatorname{Inf}_{f}(i)>0.1$.

Proof. Let $f$ be a random function over $k$ variables. The influence of some variable $i$ is determined by the number of pairs of inputs, which differ only on the coordinate $i$, that disagree on the output. Since each output of the function is chosen independently, and as these $2^{k-1}$ pairs are disjoint, this is in fact a binomial random variable. The influence of variable $i$ is less than 0.1 only if at most $1 / 5$ of these pairs disagree. This probability is thus

$$
\operatorname{Pr}\left[B\left(2^{k-1}, 0.5\right)<\frac{1}{5} \cdot 2^{k-1}\right]<2^{-c 2^{k}}
$$

for some absolute constant $c>0$, where here B is the binomial distribution and we applied one of the standard estimates for binomial distributions (cf., e.g. [3], Appendix A). Therefore, by the union bound, all $\mathrm{k}$ variables have influence greater than 0.1 with probability $1-2^{-\Omega(k)}$.

Proposition 2.3. Let $f: \mathbb{Z}_{2}^{k} \rightarrow \mathbb{Z}_{2}$ be a random core of a $k$-junta. Then with probability at least $1-2^{-\Omega(k)}, f$ is 0.1-far from any non-trivial isomorphic function.

Proof. Let $f$ be a random function of $k$ variables and let $\pi \in \mathcal{S}_{k}$ be any non-trivial permutation. Our goal is to show that $\operatorname{Pr}_{x}\left[f(x) \neq f_{\pi}(x)\right]>0.1$ for every such $\pi$, with high probability (where the probability is over the choice of $f$ and applies to all permutations simultaneously). We will do a similar calculation to the one above. Here however, we do not have a nice partition of the inputs into disjoint pairs, as some inputs remain unchanged after applying the permutation. Consider the partition of the inputs according to $\pi$ into chains, that is elements $x, \pi x, \pi^{2} x, \ldots, \pi^{i} x=x$. Notice that if $\pi$ is not the identity, there are at most $2^{k} / 2$ chains of length 1 (half of the elements). Looking at the elements of a chain of length $i \geq 2, i-1 \geq\lceil i / 2\rceil$ of them result in pairs $x, \pi x$ so that all these events $f(x) \neq f(\pi x)$ are mutually independent. Thus in total we have at least $2^{k} / 4$ independent samples.

As before, we can now bound the probability that $\operatorname{Pr}_{x}\left[f(x) \neq f_{\pi}(x)\right]<0.1$ by the probability that at most $2 / 5$ of these pairs would disagree (as with probability at least $1 / 4$ we fall into an element from our independent samples). We bound this probability by

$$
\operatorname{Pr}\left[B\left(2^{k} / 4,0.5\right)<\frac{2}{5} \cdot \frac{2^{k}}{4}\right]<2^{-c^{\prime} 2^{k}}
$$

for some absolute constant $c^{\prime}>0$, where again we applied one of the standard estimates for binomial distributions. Since there are only $k !-1$ non-trivial permutations, we can apply the union bound and conclude that over the choice of $f$, it is 0.1-far from all its non-trivial isomorphic copies with probability at least $1-2^{-\Omega(k)}$.

\subsection{Finding the influencing sets}

In order to find the influencing sets, we first need a way to estimate the influence of a set by querying the input function. To this end we use the following natural algorithm Estimate-InfLuence. 


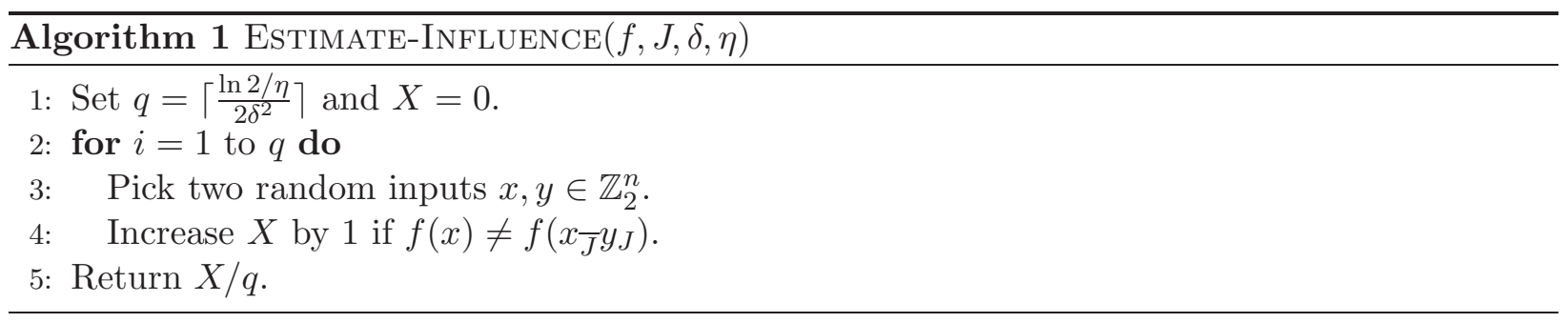

Proposition 2.4. For any function $f$, a set of variables $J$ and two constants $\delta, \eta \in(0,1)$, the algorithm Estimate-Influence $(f, J, \delta, \eta)$ returns a value within distance $\delta$ of $\operatorname{Inf}_{f}(J)$ with probability at least $1-\eta$, by performing $O\left(\delta^{-2} \log 1 / \eta\right)$ non-adaptive queries to $f$.

Proof. The proof is a direct application of the Chernoff bound as $\mathbf{E}[X / q]=\operatorname{Inf}_{f}(J)$ and we deviate by more than $\delta$ with probability at most $2 \exp \left(-2 \delta^{2} q\right)$.

In our scenario, however, we also need to consider the fact that we query a noisy version of the function. The following proposition shows that the noise cannot modify the influence of a set by too much, and therefore we can still estimate correctly which sets have significant influence.

Proposition 2.5. Let $f$ and $g$ be any two functions which are $\varepsilon$-close. Then for every set $J \subseteq[n]$ of variables, $\left|\operatorname{Inf}_{f}(J)-\operatorname{Inf}_{g}(J)\right| \leq 2 \varepsilon$.

Proof. Fix $J$ to be some subset of the variables and let $f$ and $g$ be two functions which are $\varepsilon$-close. We prove that $\operatorname{Inf}_{f}(J) \leq \operatorname{Inf}_{g}(J)+2 \varepsilon$, from which the proposition follows by simply replacing the roles of $f$ and $g$. By the triangle inequality,

$$
\begin{aligned}
\operatorname{Inf}_{f}(J) & =\operatorname{Pr}_{x, y}\left[f(x) \neq f\left(x_{\bar{J}} y_{J}\right)\right] \\
& \leq \operatorname{Pr}_{x}[f(x) \neq g(x)]+\underset{x, y}{\operatorname{Pr}}\left[g(x) \neq g\left(x_{\bar{J}} y_{J}\right)\right]+\underset{x, y}{\operatorname{Pr}}\left[g\left(x_{\bar{J}} y_{J}\right) \neq f\left(x_{\bar{J}} y_{J}\right)\right] \\
& \leq \operatorname{Inf}_{g}(J)+2 \varepsilon .
\end{aligned}
$$

We are now ready to describe the first step in our algorithm for local correction of juntas, which is identifying the influencing sets. A crucial restriction we must assume over the function we try to correct is that the influence of any influencing variable is significant enough, so we can identify them in spite of the noise (namely, it should satisfy Proposition 2.2).

The algorithm Find-INFLUENCING-SETS is given a function $f$, a partition of the variables $\mathcal{I}$, a size parameter $k$ and a noise parameter $\varepsilon$. The algorithm returns all parts in the partition $\mathcal{I}$ which it considered as influential with respect to $\varepsilon$. We will later see that in the scenario we apply it, the returned sets are exactly those which contain significantly influencing variables of $f$, with high probability.

Remark. The algorithm FIND-INFLUENCING-SETS is very similar to the algorithm BLOCKTEST defined in [5]. The main difference is in the noise tolerance behavior. In the original algorithm a part $I_{j}$ was marked as non influential, and was removed from $S$, only if its estimated influence was precisely 0. Here however we mark such a part as non-influential even if it has some influence, but as long as our estimate for it is small enough. 


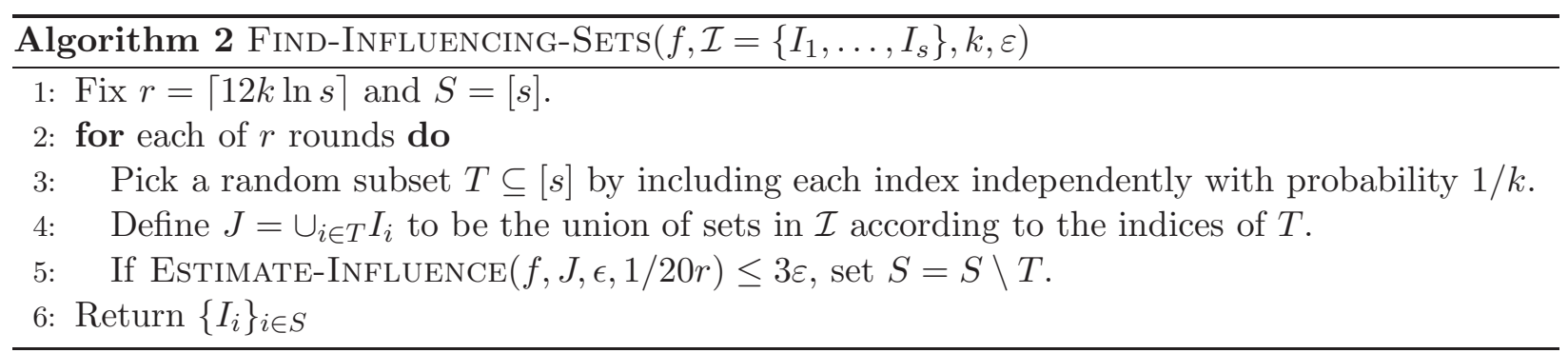

Lemma 2.6. Let $f$ be a $k$-junta whose influencing variables each has influence of at least $6 \varepsilon$ for some $\varepsilon>0$, and they are separated by a partition $\mathcal{I}$, of size $|\mathcal{I}|=O\left(k^{2}\right),|\mathcal{I}|>5$. Then for every function $g$ which is $\varepsilon$-close to $f$, Find-INFLUENCING-SETS $(g, \mathcal{I}, k, \varepsilon)$ returns exactly the $k$ sets containing the influencing variables of $f$ with probability at least $9 / 10$, by performing $O\left(k \log ^{2} k / \varepsilon^{2}\right)$ non-adaptive queries to $g$.

Proof. Fix $\varepsilon>0$ and let $f, k, \mathcal{I}$ and $g$ be as described in the lemma. We first note that the query complexity is indeed $O\left(k \log ^{2} k / \varepsilon^{2}\right)$ as we have $r=O(k \log s)=O(k \log k)$ rounds, assuming $s=O\left(k^{2}\right)$, and in each round we perform $O\left(\log s / \varepsilon^{2}\right)=O\left(\log k / \varepsilon^{2}\right)$ queries. Moreover, the queries are all non-adaptive as we only apply the EsTIMATE-INFLUENCE algorithm which is non-adaptive as well.

By the analysis of Estimate-Influence and the parameters we provide it, we know it would deviate by more than $\varepsilon$ with probability at most $1 / 20 \mathrm{r}$. Since we invoke it once per round and there are only $r$ rounds, by the union bound they would all deviate by at most $\varepsilon$ simultaneously with probability at least 19/20. Assuming this is indeed the case, what remains to be shown is that every set containing an influencing variable would be returned, and only those.

Let $I$ be a set containing an influencing variable of $f$. Since we know each influential variable of $f$ has influence at least $6 \varepsilon$, by monotonicity of influence we have $\operatorname{Inf}_{f}(J) \geq \operatorname{Inf}_{f}(I) \geq 6 \varepsilon$ for any set $J$ such that $I \subseteq J$. Moreover, since $g$ is $\varepsilon$-close to $f$, by Proposition 2.5 we have $\operatorname{Inf}_{g}(J) \geq 4 \varepsilon$ for any such set $J$. As we assumed all calls to Estimate-INFLuence deviated by at most $\varepsilon$, we would not flag the set $I$ as non-influential at any round (and thus it would be returned).

Consider now the case that $I$ contains no influencing variable of $f$. It suffices to show that at some round, the set $J$ would contain $I$ but no other set $I^{\prime}$ which contains an influencing variable. If there was such a round, then $\operatorname{Inf}_{g}(J) \leq \operatorname{Inf}_{f}(J)+2 \varepsilon=2 \varepsilon$ and we would estimate it correctly to be at most $3 \varepsilon$ by our assumption. Since there are at most $k$ sets with influencing variables, at each round, $J$ would include $I$ and no other set with influencing variables with probability at least $(1 / k)(1-1 / k)^{k} \geq 1 / 4 k$ for $k \geq 2$. We can now bound the probability that the set $I$ would be incorrectly returned by $(1-1 / 4 k)^{r} \leq e^{-r / 4 k} \leq e^{-3 \ln s}<\frac{1}{20 s}$. By applying the union bound over all the parts in $\mathcal{I}$, we get that with probability at least $19 / 20$, all sets without influencing variables would not be returned.

Combining both our assumptions, each occurring with probability at least 19/20, we indeed showed that with probability at least $9 / 10$, precisely the sets which contain the influencing variables of $f$ would be returned. 


\subsection{The algorithm}

Before we proceed to describe the complete algorithm, we need some additional definitions. Since we have no intention to identify the exact location of the influencing variables, and we only identify sets which contain them, it is natural to query the function at inputs which are constant over the sets in a given partition. For this purpose, we use the following distribution.

Definition 2.7. Let $\mathcal{I}=\left\{I_{1}, \ldots, I_{2 s}\right\}$ be a partition of $[n]$ into an even number of parts (where some parts may be empty). The distribution $\mathcal{D}_{\mathcal{I}}$ over $y \in \mathbb{Z}_{2}^{n}$ is defined as follows.

- Choose $z \in \mathbb{Z}_{2}^{2 s}$ to be a random balanced vector of Hamming weight $|z|=s$.

- Define $y \in \mathbb{Z}_{2}^{n}$ such that for every $I_{j} \in \mathcal{I}$ and $i \in I_{j}, y_{i}=z_{j}$.

Proposition 2.8 ([10]). Let $J=\left\{j_{1}, \ldots, j_{k}\right\} \subseteq[n]$ be a set of size $k$, and let $s=\Omega\left(k^{2}\right)$ be even. The distribution $\mathcal{D}_{\mathcal{I}}$ satisfies the following conditions.

- For every $x \in \mathbb{Z}_{2}^{n}, \operatorname{Pr}_{\mathcal{I}, y \sim \mathcal{D}_{\mathcal{I}}}[y=x]=2^{-n}$ given that the partition $\mathcal{I}$ was chosen at random.

- The marginal distribution of y over the set of indices $J$ is $4 k^{2} /$ s-close to uniform over $\mathbb{Z}_{2}^{k}$ (in total variation distance), for a fixed partition $\mathcal{I}$ which separates the variables of $J$.

Remark. In our scenario, we sometimes use the distribution $\mathcal{D}_{\mathcal{I}_{0} \cup \mathcal{I}_{1}}$ where $\mathcal{I}_{0}$ and $\mathcal{I}_{1}$ are random partitions of $X_{0}$ and $X_{1}$, such that $\left|\mathcal{I}_{0}\right|=\left|\mathcal{I}_{1}\right|=2 s$, and where $X_{0} \cup X_{1}=[n]$ are a partition of $[n]$ into two parts. We define $y \sim \mathcal{D}_{\mathcal{I}_{0} \cup \mathcal{I}_{1}}$ to be a merge of $y_{0} \sim \mathcal{D}_{\mathcal{I}_{0}}$ and $y_{1} \sim \mathcal{D}_{\mathcal{I}_{1}}$ in the following way. The vector $y$ is the unique vector for which $y_{X_{0}}=y_{0}$ and $y_{X_{1}}=y_{1}$. Thus, the first item of Proposition 2.8 holds as is, and in the second item we have an additional factor of 2.

The full algorithm is described as Algorithm 3 below. From this point on, whenever we draw a random partition of some set, we do so by assigning each element into one of the parts uniformly and independently at random (which may result in some empty parts). Recall that our goal is to locally correct a given function $g$, which is $\varepsilon$-close to $f_{\sigma}$, by returning the value $f_{\sigma}(x)$ for the given input $x$. Additionally, $f$ is a $k$-junta whose core is known to the algorithm, and we can and will restrict ourselves to such functions which satisfy typical conditions (namely Propositions 2.2 and 2.3). Notice that in our scenario, the smaller $\varepsilon$ is the easier it is to correct so it suffices to show that for $\varepsilon=0.001$, the algorithm succeeds with good probability and with the requested query complexity.

Proof of Theorem 1.1. First, we analyze the query complexity of the algorithm. All the queries the algorithm perform are by invoking FIND-INFLUENCING-SETS and querying $y$ which was chosen according to $\mathcal{D}_{\mathcal{I}}$. In both cases, the queries only depend on the partitions $\mathcal{I}_{0}$ and $\mathcal{I}_{1}$, and therefore they are non-adaptive. The number of queries in these parts are $O\left(k \log ^{2} k\right)$ and $O(k \log k)$ respectively, and thus the algorithm performs a total of $O\left(k \log ^{2} k\right)$ non-adaptive queries as required.

Let $f$ be a function which satisfies the conditions of Propositions 2.2 and 2.3. As each condition is satisfied with probability at least $1-2^{-\Omega(k)}$, it suffices to show the algorithm succeeds with high probability for such functions.

The success of the algorithm depends on the following three events. The first, we need the partition $\mathcal{I}$ to separate all the junta's influencing variables. In each set of variables, $X_{0}$ and $X_{1}$, 


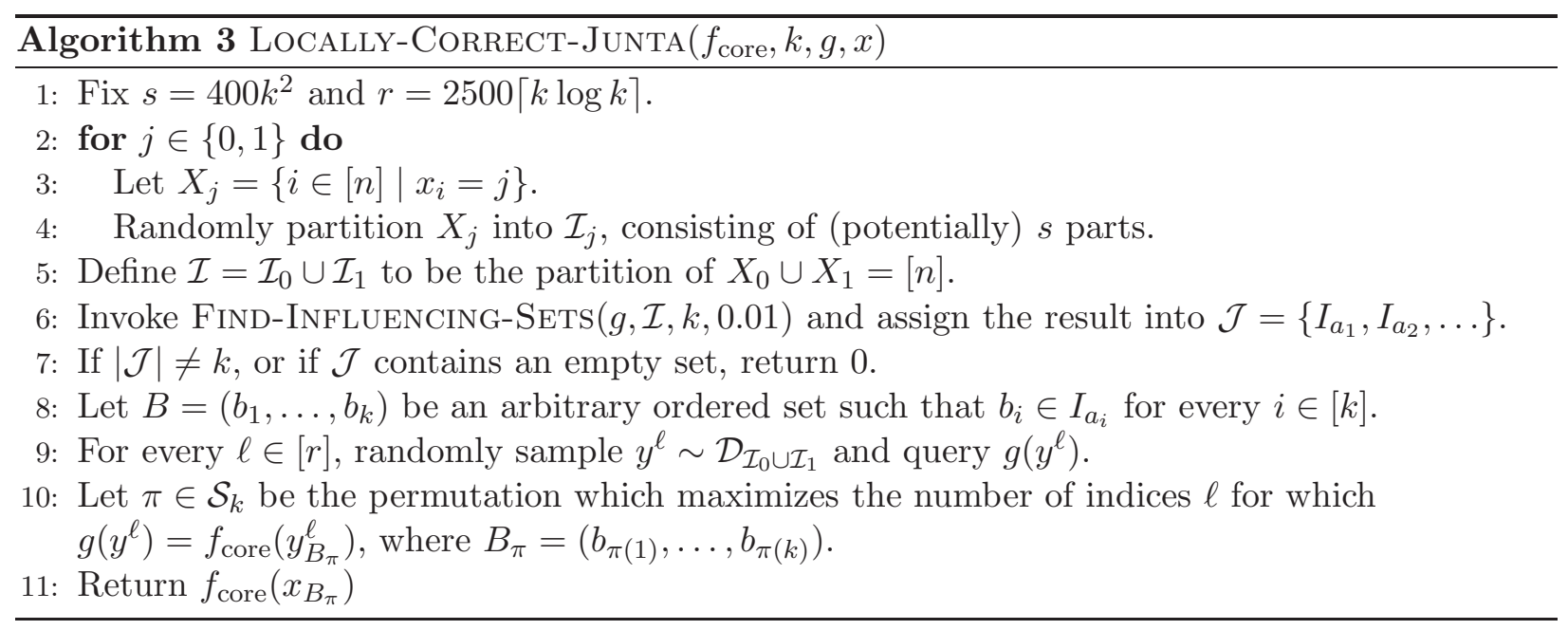

there are at most $k$ influencing variables. Since we partition each of them into $s$ parts at random, we would have such a bad collision with probability at most $2\left(\begin{array}{c}k \\ 2\end{array}\right) / s<1 / 30$ for our choice of $s$.

The second event is identifying the correct sets, those for which $f_{\sigma}$ has influencing variables. Assuming there were no collisions in the partition, by Lemma 2.6 we will identify precisely the influencing sets with probability at least 9/10.

The third and last event which remains is correctly choosing the permutation $\pi$. Here we rely on the fact that the core of $f$ is not close to any non trivial permutation of itself. Since our estimates are performed using queries according to $\mathcal{D}_{\mathcal{I}_{0} \cup \mathcal{I}_{1}}$, we need to estimate how accurate they are. By proposition 2.8, as each sample $y$ is distributed uniformly, the distance between $g(y)$ and $f_{\sigma(y)}$ is at most 0.001 over the choice of $\mathcal{I}_{0}$ and $\mathcal{I}_{1}$. By applying Markov's inequality, our partitions will satisfy $\operatorname{Pr}_{y \sim \mathcal{D}_{\mathcal{I}_{0} \cup \mathcal{I}_{1}}}\left[g(y) \neq f_{\sigma}(y)\right] \leq 0.01$ with probability at least $9 / 10$.

Assume from this point on that the partitions $\mathcal{I}_{0}$ and $\mathcal{I}_{1}$ were good, namely, satisfying the above inequality. By the second part of proposition 2.8, the marginal distribution of $y$ over the influencing variables, and hence over the influencing sets (for each partition $\mathcal{I}_{j}$ ), is $4 k^{2} / s$-close to uniform in total variation distance. Therefore, when $\pi \in \mathcal{S}_{k}$ is not the correct permutation we have

$$
\mathbf{E}_{y \sim \mathcal{D}_{\mathcal{I}_{0} \cup \mathcal{I}_{1}}}\left[g(y)=f_{\text {core }}\left(y_{B_{\pi}}\right)\right] \leq 1-0.1+0.01+2 \frac{4 k^{2}}{s}=0.93
$$

where $B_{\pi}$ are defined as in the algorithm. For the correct permutation however, this expectation would be at least 0.97. Since our queries are independent, we can apply the Chernoff bound. When we perform $r$ such queries, we deviate from the expectation by at least 0.02 with probability at most $\exp \left(-2 \cdot 0.02^{2} \cdot r\right)=\exp (-2\lceil k \log k\rceil)$. By the union bound, our estimation for all $k$ ! permutations is within 0.02 from the expectation with probability at least 9/10. Combining this with the probability that the partitions are good, it follows that we choose the correct permutation $\pi$ with probability at least $4 / 5$.

The failure probability of our algorithm can be bounded by the probability that one of the above events does not occur. Therefore, the algorithm fails with probability at most $1 / 30+1 / 10+4 / 5=$ $1 / 3$, meaning it returns the correct answer with probability at least $2 / 3$, as required.

Remark. The assertion of the theorem holds also when the core of $f$ is isomorphic to itself for some non-trivial permutation. The crucial requirement is that it is not $\varepsilon$-close to any of its permutations for $0<\varepsilon<0.1$. For simplicity, the proof does not consider these cases, however this only 
influences the identification of the permutation $\pi$ which is indifferent to which of the isomorphisms it corresponds.

\section{Correcting partially symmetric functions}

The algorithm for local correction of partially symmetric functions is similar to the one just presented for locally correcting juntas. The main tool for generalizing the algorithm and its proof is the analogous measure to influence called symmetric influence, introduced in [8]. We repeat the definition of symmetric influence and some of its properties in Section 3.1, where we also prove several properties of typical partially symmetric functions.

In Section 3.2 we describe the algorithms for estimating the symmetric influence of a set and identifying all the asymmetric sets in a partition (namely, sets which have large symmetric influence). This is the first step in the correcting algorithm. The second step, which is again recovering the ordering of the identified sets, is described as part of the algorithm in Section 3.3 , followed by the proof of Theorem 1.2 .

\subsection{Properties of partially symmetric functions}

Unlike juntas, partially symmetric functions typically depend on all the variables of the input. However, there is a large set of variables which influence the function only according to their combined Hamming weight, and not according to the value of each coordinate. The concise representation of partially symmetric functions is therefore different from that of juntas.

Let $f: \mathbb{Z}_{2}^{n} \rightarrow \mathbb{Z}_{2}$ be an $(n-k)$-symmetric function. We define the core of $f$ by $f_{\text {core }}: \mathbb{Z}_{2}^{k} \times$ $\{0,1, \ldots, n-k\} \rightarrow \mathbb{Z}_{2}$, the function $f$ restricted to its $k$ asymmetric variables and the Hamming weight of the remaining variables.

The notion of influence represents how much a variable, or a set of variables, can influence the output of the function when their value is modified. For partially symmetric functions however, as typically all variables influence the output of the function, this is less useful. Instead, as the special variables are in fact the asymmetric variables of the function, we find the following definition of symmetric influence more useful. We measure for a set of variables, what is the probability that reordering them would result in a change of the output of the function.

Definition 3.1 (Symmetric influence, [8]). Given a Boolean function $f: \mathbb{Z}_{2}^{n} \rightarrow \mathbb{Z}_{2}$, the symmetric influence of a set of variables $J \subseteq[n]$ with respect to $f$ is defined by

$$
\operatorname{SymInf}_{f}(J)=\operatorname{Pr}_{x \in \mathbb{Z}_{2}^{n}, \pi \in \mathcal{S}_{J}}[f(x) \neq f(\pi x)],
$$

where $\mathcal{S}_{J}$ is the set of permutations within $\mathcal{S}_{n}$ which only move elements inside the set $J$.

Similar to juntas, a function $f$ is $(n-k)$-symmetric if and only if there exists a set $K$ of size at most $k$ such that $\operatorname{SymInf}_{f}([n] \backslash K)=0$. The authors of [8] defined symmetric influence and showed it satisfies several properties which are similar to those of influence. One of these properties is monotonicity. For any two sets $J \subseteq K$ and any function $f, \operatorname{SymInf}_{f}(J) \leq \operatorname{SymInf}_{f}(K)$. As in the case of juntas, our algorithm heavily relies on this fact in order to identify the asymmetric sets.

Like Theorem 1.1, Theorem 1.2 is also applicable only to most partially symmetric functions and not to all of them. We again define two properties which are required for our algorithm to succeed 
with high probability, which are typical to such functions. The first bounds the symmetric influence of sets containing at least one asymmetric and one symmetric variable, presented in Proposition 3.2 . The second, described in Proposition 3.3, deals with the distance between such a function and its non-trivial isomorphisms.

Proposition 3.2. Let $f: \mathbb{Z}_{2}^{n} \rightarrow \mathbb{Z}_{2}$ be a random $(n-k)$-symmetric function for some $k<n$. Then with probability at least $1-2^{-\Omega(\sqrt{n})}$, any asymmetric variable $i$ and any symmetric variables $j$ have symmetric influence $\operatorname{SymInf}_{f}(\{i, j\})>0.1$.

Proof. Let $f$ be a random $(n-k)$-symmetric function and assume without loss of generality that its asymmetric variables are the first $k$ variables. We arbitrarily choose the asymmetric variable $x_{k}$ and the symmetric variable $x_{k+1}$. Let $x$ be a random vector and apply a random permutation on $x_{k}$ and $x_{k+1}$. Notice that only with probability $1 / 4$ we might see two different outputs of $f$, as with probability $3 / 4$ either $x_{k}=x_{k+1}$, or the chosen permutation is the identity. We therefore restrict ourselves to such inputs where $x_{k} \neq x_{k+1}$ and the permutation transposes $x_{k}$ and $x_{k+1}$.

We consider the partition of inputs to $f_{\text {core }}$ into $2^{k-1}(n-k-1)$ pairs according to the value of $x_{1}, \cdots, x_{k-1}$ and the Hamming weight of the variables $x_{k+2}, \ldots, x_{n}$. Notice that since we require $x_{k}$ to be different from $x_{k+1}$, for each such restriction we have precisely two inputs to the core. Moreover, the transposition of $x_{k}$ and $x_{k+1}$ only swaps between the two inputs of the same pair, meaning the pairs are independent. Define $m=n-k-2$ and for every $z \in \mathbb{Z}_{2}^{k-1}$ and $w \in\{0,1, \ldots, m\}$ let $X_{z, w}$ be the indicator random variable of the event that $f_{\text {core }}$ agrees on the corresponding pair of inputs. Using these definitions, we can compute the symmetric influence of $k$ and $k+1$ as follows.

$$
\operatorname{SymInf}_{f}(\{k, k+1\})=\frac{1}{8}+\frac{1}{8} \sum_{z \in \mathbb{Z}_{2}^{k-1}} \sum_{w=0}^{m} \frac{\left(\begin{array}{l}
m \\
w
\end{array}\right)}{2^{k-1} 2^{m}} \cdot(-1)^{X_{z, w}} .
$$

To bound the deviation of the symmetric influence, we additionally define for each $z$ and $w$ the random variable $Y_{z, w}=\left(\begin{array}{c}m \\ w\end{array}\right) 2^{-(k-1)} 2^{-m} \cdot(-1)^{X_{z, w}}$. The accumulated sum over $Y_{z, w}$ for every $z$ and $w$ is a martingale. When we add a specific $Y_{z, w}$ to the sum, we modify it by $\left(\begin{array}{c}m \\ w\end{array}\right) 2^{-(k-1)} 2^{-m}$ in absolute value. Therefore, by the Azuma-Hoeffding inequality,

$$
\begin{aligned}
\left.\operatorname{Pr}_{[} \operatorname{SymInf}_{f}(\{k, k+1\})<0.1\right] & \leq \operatorname{Pr}\left[\left|8 \cdot \operatorname{SymInf}_{f}(\{k, k+1\})-1\right|>\frac{1}{5}\right] \\
& \leq 2 \exp \left(-\frac{\frac{1}{5^{2}}}{2 \sum_{z, w}\left|Y_{z, w}\right|^{2}}\right) \\
& =2 \exp \left(-\frac{2^{2(k-1)} 2^{2 m}}{2 \cdot 25 \cdot 2^{k-1} \sum_{w}\left(\begin{array}{c}
m \\
w
\end{array}\right)^{2}}\right) \\
& =2 \exp \left(-\frac{2^{k}}{100} \cdot \frac{2^{2 m}}{\left(\begin{array}{c}
2 m \\
m
\end{array}\right)}\right) \\
& \approx 2 \exp \left(-\frac{2^{k}}{100} \cdot \sqrt{\pi m}\right)=2^{-\Omega\left(2^{k} \sqrt{n-k}\right)},
\end{aligned}
$$

where we used the known fact $\sum_{i=0}^{m}\left(\begin{array}{c}m \\ i\end{array}\right)^{2}=\left(\begin{array}{c}2 m \\ m\end{array}\right) \approx \frac{2^{2 m}}{\sqrt{\pi m}}$.

In order to complete the proof, we apply the union bound over the $k$ possible choices for the asymmetric variable. Notice that for the symmetric variables it suffices to consider a single choice, as they are symmetric. Thus the probability that any such symmetric influence would be smaller than 0.1 is bounded by $k \cdot 2^{-\Omega\left(2^{k} \sqrt{n-k}\right)}=2^{-\Omega(\sqrt{n})}$ as required. 
Proposition 3.3. Let $f: \mathbb{Z}_{2}^{n} \rightarrow \mathbb{Z}_{2}$ be a random $(n-k)$-symmetric function for some $k<n$, and let $J$ be the set of its asymmetric variables. Then with probability at least $1-2^{-\Omega(\sqrt{n})}, f$ is 0.1 -far from any non-trivial permutation of itself which only moves elements within $J$.

Proof. Let $f$ be a random $(n-k)$-partially symmetric function and assume without loss of generality that its asymmetric variables are the first $k$ variables. Our function $f$ is in fact a union of $n-k+1$ randomly chosen $k$-juntas over the first $k$ variables, where the Hamming weight of the remaining varialbes determines which junta we are invoking. When $k \geq \sqrt{n}$, we can apply Proposition 2.3 over each of these $k$-juntas. Each such function is 0.1-far from being isomorphic to itself with probability at least $1-2^{-\Omega(k)}$, and we apply the union bound over them. Therefore, $f$ would be 0.1-far from any non-trivial isomorphism of itself, which only moves elements within the first $k$ variables, with probability at least $1-(n-k+1) \cdot 2^{-\Omega(k)}=1-2^{-\Omega(\sqrt{n})}$.

Assume now that $k<\sqrt{n}$, and moreover that $k \geq 2$ (as when $k=0$ and $k=1$ the proposition trivially holds). Let $f_{0}, \ldots, f_{n-k+1}$ denote the $k$-juntas representing $f$. We fix some permutation $\pi \in \mathcal{S}_{k}$ over the first $k$ variables. As in the proof of Proposition 2.3. for every fixed $w$ there are at least $2^{k} / 4$ pairs of inputs $x, \pi x \in \mathbb{Z}_{2}^{k}$ for which the values of the events $f_{w}(x) \neq f_{w}(\pi x)$ are independent. By applying the Azuma-Hoeffding inequality as before for such an input $x$, we have

$$
\operatorname{Pr}\left[\mathbf{E}_{w \sim B(n-k, 1 / 2)}\left[f_{w}(x) \neq f_{w}(\pi x)\right]<0.45\right]<2^{-\Omega(\sqrt{n-k})}=2^{-\Omega(\sqrt{n})} .
$$

The probability of these events is very small, and therefore over our independent $2^{k} / 4$ samples we would have no more than $2^{k} / 40$ of them occur, with probability at least $1-2^{-\Omega\left(\sqrt{n} \cdot 2^{k}\right)}$. So overall for a given permutation $\pi$, the distance between $f$ and $f_{\pi}$ is at least $\frac{1}{4} \cdot \frac{9}{10} \cdot 0.45>0.1$, with this probability.

To complete the proof, we apply the union bound over the $k$ ! permutations, and conclude that with probability at least $1-k ! \cdot 2^{-\Omega\left(\sqrt{n} \cdot 2^{k}\right)}>1-2^{-\Omega(\sqrt{n})}$ all distances would be at least 0.1 as required.

\subsection{Finding the asymmetric sets}

The asymmetric sets in the partition are those with non-zero symmetric influence. The following algorithm estimates the symmetric influence of a given set efficiently.

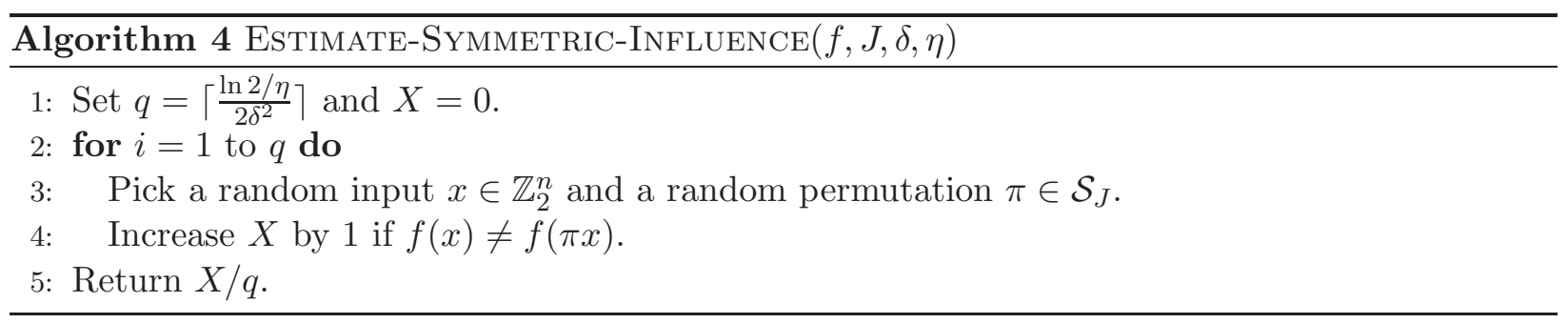

Proposition 3.4. For any function $f$, a set of variables $J$ and two constants $\delta, \eta \in(0,1)$, the algorithm Estimate-Symmetric-Influence $(f, J, \delta, \eta)$ returns a value within distance $\delta$ of $\operatorname{SymInf}_{f}(J)$ with probability at least $1-\eta$, by performing $O\left(\delta^{-2} \log 1 / \eta\right)$ non-adaptive queries to $f$.

Proof. The proof is a direct application of the Chernoff bound as $\mathbf{E}[X / q]=\operatorname{SymInf}_{f}(J)$ and we deviate by more than $\delta$ with probability at most $2 \exp \left(-2 \delta^{2} q\right)$. 
Since our goal is to identify the asymmetric sets in the presence of noise, we rely on the following proposition. Similar to influence, if two functions are $\varepsilon$-close, the symmetric influence of every set deviates by at most $2 \varepsilon$. We omit the proof as it is identical to that of Proposition 2.5.

Proposition 3.5. Let $f$ and $g$ be any two functions which are $\varepsilon$-close. Then for every set $J \subseteq[n]$ of variables, $\left|\operatorname{SymInf}_{f}(J)-\operatorname{SymInf}_{g}(J)\right| \leq 2 \varepsilon$.

The first part in correcting partially symmetric functions is identifying the asymmetric sets. This algorithm is identical to the one for identifying the influencing sets in juntas, with the only difference of invoking Estimate-SymmetriC-INFLUENCE instead of Estimate-INFLuence. We require our input function to satisfy the condition of Proposition 3.2 in order to correctly identify the asymmetric sets in spite of the noise.

The algorithm Find-AsymmetriC-SETs is given a function $f$, a partition of the variables $\mathcal{I}$, a size parameter $k$ and a noise parameter $\varepsilon$. The algorithm returns all parts in the partition $\mathcal{I}$ which it considered as asymmetric with respect to $\varepsilon$.

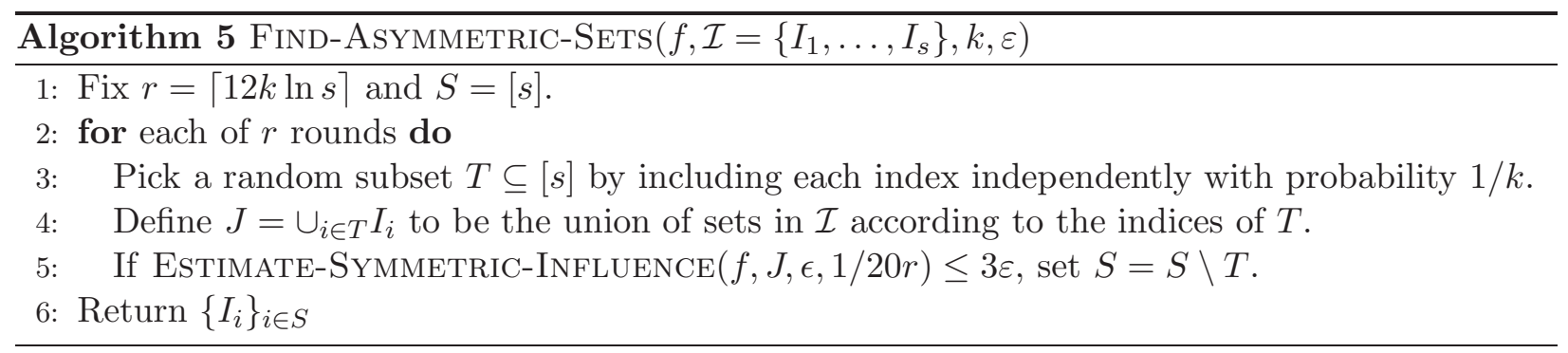

Lemma 3.6. Let $f$ be an $(n-k)$-symmetric function for $k=o(n / \log n)$, which satisfies the following conditions. The asymmetric variables of $f$ are separated by the partition $\mathcal{I}$, which is of size $|\mathcal{I}|=O\left(k^{2}\right),|\mathcal{I}|>5$. Additionally, the symmetric influence of every asymmetric variable and a symmetric variable is at least $6 \varepsilon$ for some $\varepsilon>0$. Then for every function $g$ which is $\varepsilon$-close to $f$, Find-AsymmetriC-SETS $(g, \mathcal{I}, k, \varepsilon)$ returns exactly the $k$ sets containing the asymmetric variables of $f$ with probability at least $9 / 10$, by performing $O\left(k \log ^{2} k / \varepsilon^{2}\right)$ non-adaptive queries to $g$.

Proof. The proof of the above lemma is identical to that of Lemma 2.6, where we replace influence with symmetric influence when applicable. Proposition 3.5 guarantees that in the noisy function $g$ we would still be able to distinguish the asymmetric sets. Additionally, by the limitation over $k$, with high probability in all rounds we would have at least one symmetric variable, which guarantees the high symmetric influence (assuming there is also an asymmetric variable).

\subsection{The algorithm}

The second step of the algorithm is recovering the correct order of the $k$ asymmetric sets. As explained before, it is reasonable to query the input function over inputs which are constant over the various parts of our random partition. In this case however, we also care about the Hamming weight of the inputs we query. Due to this, we cannot allow our queries to be consistent over all the parts, but rather we will dedicate one part for adjusting the distribution of the Hamming weight. We name this special part workspace, and we choose it arbitrarily from our random partition.

Definition 3.7. Let $\mathcal{I}$ be some partition of $[n]$ into an odd number of parts and let $W \in \mathcal{I}$ be the workspace. Define the distribution $\mathcal{D}_{\mathcal{I}}^{W}$ over $\mathbb{Z}_{2}^{n}$ to be as follows. Pick a random Hamming 
weight $w$ according to the binomial distribution $B(n, 1 / 2)$ and output, if exists, a random $x \in \mathbb{Z}_{2}^{n}$ of Hamming weight $|x|=w$ such that for every $I \in \mathcal{I} \backslash\{W\}$, either $x_{I} \equiv 0$ or $x_{I} \equiv 1$. When no such $x$ exists, return the all zeros vector.

The above distribution, together with the random choice of the partition and workspace, satisfies the following two important properties. The first, being close to uniform over the inputs of the function. The second, having a marginal distribution over the inputs to the core of a partially symmetric function close to typical. A typical distribution over the core of an $(n-k)$-symmetric function is the product of a uniform distribution over $\mathbb{Z}_{2}^{k}$ and $B(n-k, 1 / 2)$. These properties are formally written here as Proposition 3.8 which originally appeared in $[8]$.

Proposition $3.8([8])$. Let $J=\left\{j_{1}, \ldots, j_{k}\right\} \subseteq[n]$ be a set of size $k$, and $s=\Omega\left(k^{2}\right)$ be odd. If $y \sim \mathcal{D}_{\mathcal{I}}^{W}$ for a random partition $\mathcal{I}$ of $[n]$ into $s$ parts and a random choice of the workspace $W \in \mathcal{I}$, then

- y is o(1/n)-close to being uniform over $\mathbb{Z}_{2}^{n}$, and

- $\left(y_{J},\left|y_{\bar{J}}\right|\right)$ is $(k / s+o(1))$-close to being distributed uniformly over $\mathbb{Z}_{2}^{k}$ and binomial over $\{0,1, \ldots, n-k\}$, for a fixed partition $\mathcal{I}$ which separates the variables of $J$.

Remark. Our algorithm will choose the partition $\mathcal{I}$ and workspace $W$ not entirely at random, but rather it will consider the location of zeros and ones in the input $x$. However, the above proposition still holds in this scenario, assuming we partition each of the two sets into $r$ parts at random.

We can now describe the full algorithm, followed by its analysis. Recall that our goal is to locally correct a given function $g$, which is $\varepsilon$-close to $f_{\sigma}$, by returning the value $f_{\sigma}(x)$ for the given input $x$. Additionally, $f$ is an $(n-k)$-symmetric function whose core is known to the algorithm, and we can and will restrict ourselves to such functions which satisfy typical conditions (namely Propositions 3.2 and 3.3). Notice that in our scenario, the smaller $\varepsilon$ is the easier it is to correct so it suffices to show that for $\varepsilon=0.001$, the algorithm succeeds with good probability and with the requested query complexity.

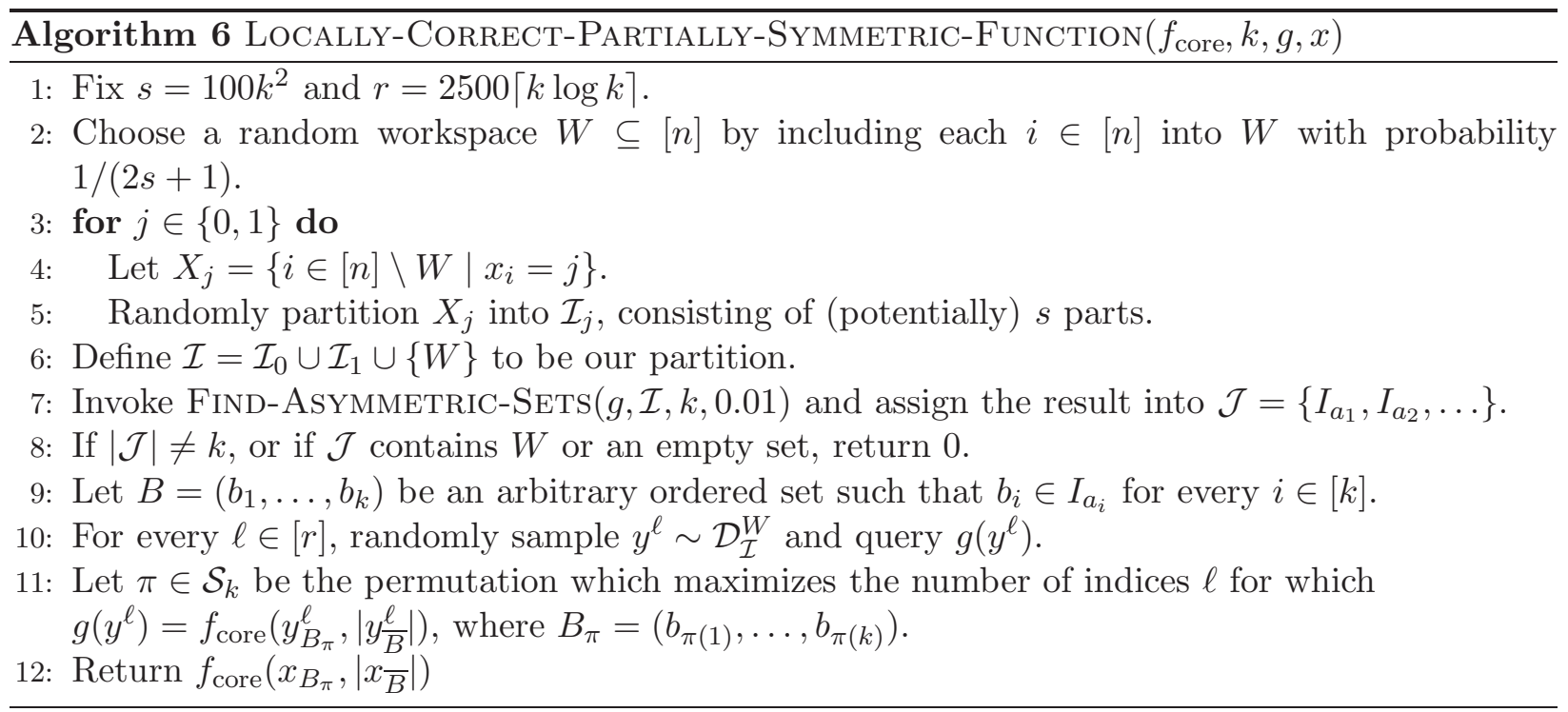


Proof of Theorem 1.2. The queries of the algorithm are performed by invoking FInD-AsYMmETRICSETS and by sampling according to $\mathcal{D}_{\mathcal{I}}^{W}$. In both cases the queries are non-adaptive and depend only on our choice of the partition $\mathcal{I}$ and workspace $W$. The total number of queries performed is $O\left(k \log ^{2} k\right)$, as required.

Let $f$ be an $(n-k)$-symmetric function which satisfies the conditions of Propositions 3.2 and 3.3 . As each condition is satisfied with probability at least $1-2^{-\Omega(\sqrt{n})}$, it suffices to show the algorithm succeeds with high probability when applied to these functions. Moreover, when $k=\Omega(n / \log n)$, Theorem 1.2 trivially holds by applying the standard non-adaptive algorithm of querying $O(n \log n)$ uniform queries (given that Proposition 3.3 is satisfied). Therefore, we assume $k=o(n / \log n)$ throughout the rest of the proof.

The success of the algorithm depends on the following three events. First, we need the partition $\mathcal{I}$ to separate all the asymmetric variables, and that none of them would belong to the workspace. An asymmetric variable would be chosen to the workspace with probability at most $\mathrm{k} / \mathrm{s}$. At each set of variables, $X_{0}$ and $X_{1}$, there are at most $k$ asymmetric variables and therefore each would have a collision with probability at most $\left(\begin{array}{c}k \\ 2\end{array}\right) / s$. Therefore, we would have a bad partition and workspace with probability at most $\left(k+2\left(\begin{array}{l}k \\ 2\end{array}\right)\right) / s<1 / 30$ for our choice of $s$.

The second event is identifying the correct sets, those for which $f_{\sigma}$ has asymmetric variables. Assuming the first event occurred, by Lemma 3.6 we will identify precisely the asymmetric sets with probability at least $9 / 10$.

The third and last event which remains is correctly choosing the permutation $\pi$. Here we rely on the fact that the core of $f$ is not close to any non trivial permutation of itself. Since our estimates are performed using queries according to $\mathcal{D}_{\mathcal{I}}^{W}$, we need to estimate how accurate they are. By proposition 3.8, as each sample $y$ is distributed $o(1 / n)$-close to uniform, the distance between $g(y)$ and $f_{\sigma}(y)$ is at most $0.001+o(1 / n)$ over the choice of $\mathcal{I}$ and $W$. By applying Markov's inequality, our partition and workspace will satisfy $\operatorname{Pr}_{y \sim \mathcal{D}_{\mathcal{I}}^{W}}\left[g(y) \neq f_{\sigma}(y)\right] \leq 0.02$ with probability at least $9 / 10$.

Assume from this point on that the partition and workspace satisfy the above inequality. By the second part of proposition 3.8, the marginal distribution of $y$ over the asymmetric variables (and in particular over the asymmetric sets) would be 0.01-close to uniform in total variation distance. Therefore, when $\pi \in \mathcal{S}_{k}$ is not the correct permutation we have

$$
\mathbf{E}_{y \sim \mathcal{D}_{\mathcal{I}}^{W}}\left[g(y)=f_{\text {core }}\left(y_{B_{\pi}},\left|y_{\bar{B}}\right|\right)\right] \leq 1-0.1+0.02+0.01=0.93
$$

where $B$ and $B_{\pi}$ are defined as in the algorithm. For the correct permutation, however, this expectation is at least 0.97. Since our queries are independent from one another, we can apply the Chernoff bound. When performing $r$ such queries, we deviate from the expectation by at least 0.02 with probability at most $\exp \left(-2 \cdot 0.02^{2} \cdot r\right)=\exp (-2\lceil k \log k\rceil)$. By the union bound, our estimation for all $k$ ! permutations is within 0.02 from the expectation with probability at least $9 / 10$. Combining this with the probability that the partition and workspace are good, the correct permutation $\pi$ is chosen with probability at least $4 / 5$.

The failure probability of our algorithm can be bounded by the probability that one of the above events will not occur. Therefore, the algorithm will fail with probability at most $1 / 30+1 / 10+4 / 5=$ $1 / 3$, meaning it will return the correct answer with probability at least $2 / 3$, as required.

Remark. As in Theorem 1.1, this proof also holds for partially symmetric functions which do have some non-trivial isomorphisms, but for simplicity we do not consider this here. 


\section{Conclusions and open problems}

In the previous sections we have shown that most juntas and partially symmetric functions can be efficiently locally corrected from a constant error rate. Although Proposition 1.3 indicates that not every junta or partially symmetric function satisfy this, it provides no insight on whether other functions can be locally corrected efficiently under similar conditions.

To better understand the question of which functions can be locally corrected efficiently from a constant noise rate, we present the proof of Theorem 1.4. This theorem does not provide a characterization of these locally correctable functions, but rather indicates that most functions do not fall into this category. In order to prove the theorem, we use the main results of [1, where it is shown that testing isomorphism to almost every function requires at least a linear number of non-adaptive queries.

Proposition 4.1 ([1]). For almost every Boolean function $f$ the following holds. Let $Q=Q_{b} \cup Q_{u}$ be a set of $\frac{n}{100}$ balanced queries (of Hamming weight between $\frac{n}{3}$ and $\frac{2 n}{3}$ ) and $\frac{n}{100}$ unbalanced queries. Over the choice of a random isomorphism $f_{\sigma}$ of $f$, with probability $1-o(1)$ over the output of $f_{\sigma}$ at $Q_{u}$, every possible outcome $r \in \mathbb{Z}_{2}^{\left|Q_{b}\right|}$ of querying $f_{\sigma}$ at $Q_{b}$ is obtained with probability $\left(1 \pm \frac{1}{3}\right) 2^{-\left|Q_{b}\right|}$.

Proof of Theorem 1.4. The above proposition is essentially everything needed for completing the proof. Given a random Boolean function which satisfies the condition of the above proposition, we can request one to locally correct some arbitrary balanced input. Let us assume there exists an algorithm that can perform this task with good probability, using less than $\frac{n}{100}$ non-adaptive queries. We can extend its query set to consist of $\frac{n}{100}$ balanced and $\frac{n}{100}$ unbalanced queries, including the input we asked to correct (obviously this cannot reduce its success probability).

By the above proposition, with high probability over the output of the function over the unbalanced queries, the distribution of the function over the balanced queries is very close to uniform. In fact, for every possible output over all the balanced queries except the questioned input, the marginal distribution that remains is $1 / 3$-close to uniform, and hence one would not be able to predict its value with probability $\geq 2 / 3$. Notice that for the purpose of the analysis, we assume no noise was used, where in practice we simply override the value of the questioned input with the value 0 .

Like juntas and partially symmetric functions, there are several other families it would be interesting to investigate with respect to local correction. One natural family is that of low degree polynomials. It was already shown (see [4, 2]) that these can be locally corrected using an exponential number of queries, when the noise rate is also exponentially small (in term of the degree of the polynomial). However, whether this can be done when the noise rate is constant is yet unknown.

Question. Fix some constant $\varepsilon>0$ and a degree $d>\left\lceil\log _{2} 1 / \varepsilon\right\rceil$. Do most degree $d$ polynomials over $n \geq d$ variables require $\Omega(\log n)$ queries to be locally corrected from $\varepsilon$ noise rate?

Low degree polynomials have a rather rigid structure, although they may still be far from symmetric with respect to any subset of the input variables (even for very small degrees). When the noise rate is larger than $2^{-d}$ (where $d$ is the degree of the polynomial), the noisy function can actually be another polynomial of the same degree. In such a case, making use of the structure of the polynomial seems trickier, as one cannot simply correct according to it. Given the natural 
asymmetric nature of most low degree polynomials, it may be the case that they are similar to random functions, in the sense that one needs a number of queries which grows with $n$ to perform this task, even for constant degrees.

\section{References}

[1] N. Alon and E. Blais, Testing boolean function isomorphism. In Proc. RANDOM-APPROX, pp. 394-405, 2010.

[2] N. Alon, T. Kaufman, M. Krivelevich, S. Litsyn and D. Ron, Testing low-degree polynomials over GF(2). In Proc. RANDOM-APPROX, pp. 188-199, 2003. Also: Testing Reed-Muller codes. IEEE Transactions on Information Theory 51, pp. 4032-4039, 2005.

[3] N. Alon and J. Spencer, The Probabilistic Method, Third Edition. Wiley, 2008.

[4] N. Alon and A. Weinstein, Local correction of juntas. Information Processing Letters, Volume 112, Issue 6, pp. 223-226, 2012.

[5] E. Blais, Improved bounds for testing juntas. In Proc. 12th Workshop RANDOM, pp. 317-330, 2008.

[6] E. Blais, Testing juntas nearly optimally. In Proc. 41st Annual ACM Symposium on Theory of Computing (STOC), pp. 151-158, 2009.

[7] E. Blais and R. O'Donnell, Lower bounds for testing function isomorphism. In IEEE Conference on Computational Complexity, pp. 235-246, 2010.

[8] E. Blais, A. Weinstein and Y. Yoshida, Partially symmetric functions are efficiently isomorphism-testable. In Proc. 53rd Annual IEEE Symposium on Foundations of Computer Science (FOCS), pp. 551-560, 2012.

[9] H. Chockler and D. Gutfreund, A lower bound for testing juntas. Information Processing Letters, Volume 90, Issue 6, pp. 301-305, 2004.

[10] S. Chakraborty, D. García-Soriano, and A. Matsliah, Nearly tight bounds for testing function isomorphism. In Proc. 22nd Annual ACM-SIAM Symposium on Discrete Algorithms (SODA), pp. 1683-1702, 2011.

[11] E. Fischer, G. Kindler, D. Ron, S. Safra, and A. Samorodnitsky, Testing juntas. J. Comput. Syst. Sci., Volume 68, Issue 4, pp. 753-787, 2004.

[12] Rocco A. Servedio, Testing by implicit learning: a brief survey. Property Testing, pp. 197-210, 2010 .

[13] S. Yekhanin, Locally Decodable Codes. NOW Publishers, 2010. 